\title{
Acute kidney injury after transcatheter aortic valve replacement in the elderly: outcomes and risk management
}

This article was published in the following Dove Medical Press journal:

Clinical Interventions in Aging

\author{
Marta Zaleska-Kociecka' \\ Maciej Dabrowski \\ Janina Stepinska' \\ 'Cardiac Intensive Therapy Clinic, \\ Institute of Cardiology, Warsaw, \\ Poland; ${ }^{2}$ Interventional Cardiology \\ and Angiology Clinic, Institute of \\ Cardiology, Warsaw, Poland
}

Correspondence: Marta Zaleska-Kociecka Cardiac Intensive Therapy Clinic, Institute of Cardiology, Alpejska Street 42, 04-628 Warsaw, Poland

Tel +4850l 506713

Email mzaleska@ikard.pl

\begin{abstract}
Aortic stenosis is the most common cause of valve replacement in Europe and North America with prevalence increasing with age. Transcatheter valve replacement (TAVR) represents an alternative for surgical valve replacement of severely stenotic valves. Despite lower risk of acute kidney injury compared to that associated with surgery, this complication remains prevalent in patients undergoing TAVR. There is a paucity of data confirming the relation of acute kidney injury with high morbidity and mortality, especially when superimposed on chronic kidney disease, which is a frequent comorbidity in the elderly with severe aortic stenosis. As there is no consensus on the prevention of acute kidney injury in patients undergoing TAVR, identification and limitation of risk factors are crucial. In this review, we aim to discuss the key aspects of acute kidney injury diagnosis, risk assessment, and outcomes in TAVR patients, and to point out gaps in current knowledge.
\end{abstract}

Keywords: acute kidney injury, TAVI, transcatheter valve replacement, valvular disease, aortic stenosis, aortic valve replacement

\section{Introduction}

Aortic stenosis (AS) is the most common acquired valvular disease in Europe and North America with prevalence increasing with age, thus being the most frequent cause of valve replacement. Since the advent of transcatheter valve replacement (TAVR), physicians and patients have an alternative to surgical valve replacement (SAVR). A decision on the mode of treatment is based mainly on preoperative risk assessment. ${ }^{1}$ Baseline kidney function and risk factors of perioperative acute kidney injury (AKI) are consistently included in risk scores such as EuroScore I or II, STS score, and deeply taken into consideration by physicians when determining therapeutic strategy. This is driven by a paucity of data confirming the relation of AKI with high morbidity and mortality, especially when superimposed on chronic kidney disease (CKD). ${ }^{2}$ Furthermore, we have learnt from cardiac surgery patients that even a small alteration in kidney function is related to high mortality. ${ }^{3}$ On top of that, TAVR patients mostly represent a unique population of elderly and high-risk patients prone to complications affecting their quality of life, which might be of higher importance than their lifespan. On the other hand, although originally being an alternative to SAVR in patients of prohibitive and high surgical risk (as proved in such trials as PARTNER 1A, PARTNER 1B, CORVALVE), TAVR has already been shown to be noninferior to classic surgery in the intermediate-risk population (PARTNER 2, SURTAVI). There are ongoing trials 
in low-risk patients (PARTNER 3, CorValve Evolute-R). We can expect that AKI rates and consequences will vary across different risk groups.

In this review, we aim to discuss the key aspects of AKI diagnosis, risk assessment, and outcomes in TAVR patients, and to point out gaps in current knowledge.

\section{Diagnosis and epidemiology Epidemiology}

Data on AKI prevalence in TAVR patients vary between $3.4 \%$ and $57 \%$ with $0 \%-21 \%$ requiring renal replacement therapy (RRT). ${ }^{3,4}$ Both high- and intermediate-risk patients have significantly lower AKI rates when compared to SAVR patients. ${ }^{5}$ Of note, data from German national database show an insignificant decline in the AKI rates after TAVR over time from 5.6\% in 2007 to $5.2 \%$ in 2013 with a parallel significant increase after SAVR (from $2.4 \%$ in 2007 to $3.8 \%$ in 2013). ${ }^{6}$ While improvement in outcomes after TAVR can be attributed to a "learning curve" effect, better patient selection and care, as well as advances in device development, the increase in the AKI rates after SAVR was an unexpected finding. However, it was a retrospective study, based on German Modification International Statistical Classification of Diseases without data on AKI severity, and thus should be interpreted with caution.

\section{Diagnosis}

A large discrepancy in AKI frequency is mainly driven by differences in study design and AKI definition. Since the introduction of the Valve Academic Research Criteria (VARC), AKI definition has become more unified across trials, despite the fact that the reported prevalence based on VARC is still heterogeneous and ranges from $4.6 \%$ to $35.1 \%{ }^{7}$ In agreement with the Kidney Improving Global Outcome guidelines, VARC recommends the use of AKI definition that consists of two domains, (i) creatinine increase and/or (ii) urine output volume, as displayed in Table 1 with extension of the time of observation and diagnosis up to 7 days. ${ }^{8}$ Still, the latter has been commonly neglected. A study by Shacham et al showed that AKI following the urine output criteria can constitute up to $50 \%$ of all AKI cases after TAVR. ${ }^{9}$ To date, data from randomized trials and large registries have missed urine output data. One may argue that urine output is not a reliable marker of renal insult as influenced by fluid status, but so is creatinine, thus it should not be ignored.

Creatinine, as an AKI biomarker, apart from being fluid-dependent, has several pitfalls, which are summoned up in Table 2 and were profoundly described by Osterman et al in their AKI review (see Table 2). ${ }^{10}$ In relation to the elderly and often frail TAVR patients, it is low muscle mass that affects the reliability of creatinine as an AKI indicator. Reduced creatinine production can hinder the ability of serum creatinine to reflect a true estimated glomerular filtration rate (eGFR) decrease, thus contributing to a delayed or missed diagnosis of AKI.

Last but not the least, AKI duration needs to be considered. Despite not being included in the AKI definition, duration of the kidney insult significantly contributes to patients' prognosis. The so-called persistent AKI (pAKI, with an increase in creatinine lasting up to discharge) has been shown to be related to higher mortality when it is a complication of TAVR. According to the results of the Swedish TAVI registry (SWEDEHEART), the presence of pAKI at discharge was associated with double risk of death in a 2-year follow-up compared to TAVI patients without pAKI at discharge. ${ }^{11}$

\section{Novel kidney biomarkers}

Over the last decade, the armamentarium of kidney biomarkers has expanded, yet creatinine, despite its downsides as mentioned above, is still the cornerstone of the definition and the main diagnostic tool used in clinical practice. Data on kidney biomarkers in the TAVR population are limited and do not legitimize their clinical use except for glomerular filtration cystatin C. ${ }^{12}$ Most of these promising biomarkers have either

Table I Definition and staging of acute kidney injury

\begin{tabular}{|l|l|l|}
\hline Stage & Serum creatinine $^{\text {a }}$ & Urine output \\
\hline $\mathrm{I}$ & $\begin{array}{l}1.5-1.99 \text { times baseline } \\
\text { or } \\
\geq 0.3 \mathrm{mg} / \mathrm{dL}(>+26.4 \mu \mathrm{mol} / \mathrm{L}) \text { increase }\end{array}$ & $<0.5 \mathrm{~mL} / \mathrm{kg} / \mathrm{h}$ for $6-12$ hours \\
\hline 2 & $2.0-2.99$ times baseline & $<0.5 \mathrm{~mL} / \mathrm{kg} / \mathrm{h}$ for $\geq 12 \mathrm{hours}$ \\
\hline 3 & $\begin{array}{l}3.0 \text { times baseline } \\
\text { or } \\
\text { increase in serum creatinine } \geq 4.0 \mathrm{mg} / \mathrm{dL}(\geq 354 \mu \mathrm{mol} / \mathrm{L}) \text { with an acute } \\
\text { increase of at least } 0.5 \mathrm{mg} / \mathrm{dL}(44 \mathrm{mmol} / \mathrm{L})\end{array}$ & $\begin{array}{l}<0.3 \mathrm{~mL} / \mathrm{kg} / \mathrm{h} \text { for } \geq 24 \mathrm{hours} \\
\text { or } \\
\text { anuria for } \geq 12 \text { hours }\end{array}$ \\
\hline
\end{tabular}

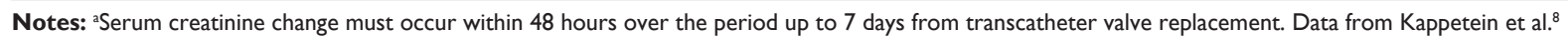


Table 2 Creatinine drawbacks as an AKI marker modified based on Ostermann et al ${ }^{10}$

\begin{tabular}{|l|l|}
\hline Clinical scenarios & Possible outcomes \\
\hline $\begin{array}{l}\text { Administration of drugs affecting tubular secretion of creatinine } \\
\text { (ie, trimethoprim) }\end{array}$ & $\begin{array}{l}\text { Misdiagnosis of AKI (increase of creatinine without alteration in } \\
\text { kidney function) }\end{array}$ \\
\hline Reduced creatinine production (ie, muscle wasting sepsis, liver disease) & Delayed or missed diagnosis of AKI \\
\hline $\begin{array}{l}\text { Intake of substances that lead to increase in creatinine generation } \\
\text { (ie, cooked meat consumption) }\end{array}$ & Misdiagnosis of AKI \\
\hline Factors affecting analytical measurement of creatinine (ie, bilirubin, cefoxitin) & Misdiagnosis or delayed diagnosis depending on the substance \\
\hline Fluid resuscitation and overload & Delayed AKI diagnosis (dilution of serum creatinine concentration) \\
\hline $\begin{array}{l}\text { Extrinsic creatinine administration as a buffer in medications } \\
\text { (ie, dexamethasone) }\end{array}$ & Pseudo-AKI \\
\hline
\end{tabular}

Note: Adapted from Ostermann M, Joannidis M. Acute kidney injury 2016: diagnosis and diagnostic workup. Crit Care. 2016;20(1):299 (https://creativecommons.org/licenses/ by/4.0/)..$^{10}$

Abbreviation: AKI, acute kidney injury.

failed or had conflicting data. Two biomarkers of tubular stress, insulin-like growth factor-binding protein 7 and the tissue inhibitor of metalloproteinases-2, were evaluated by both Zaouter et al and Dusse et al in comparably designed studies but with markedly different results. ${ }^{13,14}$ The former authors reported unsatisfactory diagnostic accuracy with areas under the receiver operating curves (AUC) varying from 0.66 to 0.71 on day 1 after procedure, while the latter found it excellent with AUC of 0.971. To date, most studies on tubular damage biomarkers (ie, NGAL) and intrarenal inflammation markers (ie, IL-18) have not yielded promising results. ${ }^{15}$ Our own data indicate that beta- 2 microglobulin reflecting glomerular filtration similar to creatinine and cystatin $\mathrm{C}$ can be a player worth attention in this population as its cutoff value at 24 hours after valve replacement reached an AUC of 0.880, outperforming cystatin C. ${ }^{16}$ In the light of the available data, early diagnosis with new biomarkers occurs to be still challenging.

\section{Risk factors}

As the pathogenesis of AKI following TAVR is multifactorial, identification of a wide variety of pre-, peri-, and postprocedural risk factors is crucial. Reported risk factors are displayed in Table 3 and consist of classic ones, common for both SAVR and TAVR, such as bleeding, as well as TAVR-specific ones, such as contrast use, hemodynamic instability on rapid ventricular pacing (RVP), or route of transcatheter valve insertion. In this review, we will mainly focus on the latter.

\section{Preprocedural Chronic kidney disease}

Most of the preprocedural AKI risk factors are patient-related, with CKD being the key player among them. The etiology of CKD is complex and is mainly attributed to chronic hemodynamic insult with low cardiac output and congestion due

Table 3 Risk factors of acute kidney injury complicating TAVR

\begin{tabular}{|c|c|c|}
\hline Preprocedural & Periprocedural & Postprocedural \\
\hline Age & Access route & Hemodynamic instability \\
\hline Chronic kidney disease & At least moderate paravalvular aortic regurgitation & $\begin{array}{l}\text { Bleeding complications/blood } \\
\text { transfusion }\end{array}$ \\
\hline $\begin{array}{l}\text { Diabetes mellitus/preprocedural glycemia } \\
\text { control }\end{array}$ & Renal embolization & Anemia \\
\hline Heart failure & Rapid ventricular pacing & $\begin{array}{l}\text { Decreased left ventricular ejection } \\
\text { fraction }\end{array}$ \\
\hline Atherosclerotic burden & Contrast media & \\
\hline \multicolumn{3}{|l|}{ COPD } \\
\hline Hydratation & Bleeding complications/blood transfusion & \\
\hline $\begin{array}{l}\text { Nephrotoxic agents (ie, nonsteroidal } \\
\text { anti-inflammatory) }\end{array}$ & $\begin{array}{l}\text { Hemodynamic instability/hypotension (need for mechanical } \\
\text { circulatory support, inotropic and vasoconstrictive drugs) }\end{array}$ & \\
\hline \multirow{2}{*}{$\begin{array}{l}\text { Global surgical risk (ie, EuroScore, } \\
\text { STS score) }\end{array}$} & Systemic inflammatory response to the procedure & \\
\hline & Conversion to open surgery & \\
\hline
\end{tabular}

Note: The TAVR-unique are bolded.

Abbreviation: TAVR, transcatheter valve replacement. 
to aortic stenosis (type 2 cardiorenal syndrome), history of diabetes mellitus, and hypertension. ${ }^{17}$ Both clinical trials and large registries report an inverse relation between eGFR and AKI risk as well as short- and long-term mortality. ${ }^{2,18}$ Despite exclusion of patients with end-stage renal disease from pivotal TAVR trials, data from registries show that over $50 \%$ of highrisk patients suffer $>3 \mathrm{a}$ CKD $(\mathrm{eGFR}<60 \mathrm{~mL} / \mathrm{kg} / \mathrm{min})$ and about $10 \%$ of them suffer $>4 \mathrm{CKD}$, and the risk of $\mathrm{AKI}$ increases significantly at each stage of CKD advancement. ${ }^{2}$ Comparing to those with SAVR, high-risk TAVR patients have significantly lower AKI rates across all CKD stages, which can improve their survival rate. ${ }^{19,20}$ At the same time, dialysis patients benefit from TAVR, having lower hospital mortality and shorter hospital stay in comparison to SAVR. ${ }^{21}$ Unfortunately, long-term results comparing the two modes of valve replacement in this population are missing. However, physicians must be aware that the 1-year mortality rate in dialysis patients undergoing TAVR exceeds $40 \%{ }^{22}$ This raises a question about the real benefit from TAVR in this population. Intermediate-risk patients also benefit from TAVR in terms of lower AKI burden when compared to SAVR patients, but their advanced-stage CKD $(>4)$ does not seem to be related to mortality. ${ }^{23}$

\section{Periprocedural}

\section{Contrast exposure}

Data on the relation between absolute contrast volume and post-TAVR AKI are inconsistent. However, some studies suggest that baseline renal function needs to be taken into consideration along with contrast volume to adequately assess insult on viable nephrons. ${ }^{24}$ As suggested by Giannini et al, patients presenting with impaired kidney function are expected to receive decreased amounts of contrast, which can cause a bias when analyzing its relation to AKI. ${ }^{25}$ In their study, there was no association between the absolute contrast volume and AKI risk after TAVR, but once normalized to baseline eGFR (contrast volume/eGFR), the absolute contrast volume turned out to be correlated not only with a higher AKI incidence but also mortality. Advanced imaging technologies, such as EchoNavigator, providing transesophageal echocardiography (TEE) and fluoroscopy fusion images, make contrast-free TAVR procedure feasible, which can be advantageous in AKI-prone patients. Despite that, a trend away from general anesthesia has limited the use of periprocedural TEE in the recent years. ${ }^{26}$ In turn, there is a dedicated tool on the market for the prevention of contrast-induced kidney injury called the RenalGuard System. This device is designed to enhance furosemide-induced high-volume diuresis with the concurrent maintenance of intravascular volume through matched hydration. It was evaluated in the PROTECT-TAVI study that reported a significantly lower AKI rate in the active treatment group (5.4\%) compared to the control group (25.2\%). The RenalGuard System has been proven to significantly reduce AKI by ensuring furosemideinduced diuresis with matched isotonic intravenous hydration during the TAVI procedure. ${ }^{27}$

\section{Hemodynamic instability}

From the pathophysiologic point of view, maintaining mean arterial pressure around $65 \mathrm{mmHg}$ is a prerequisite for the appropriate kidney perfusion and hence function. The already insufficient kidneys have disrupted autoregulation mechanisms, which make them more susceptible for further insult. Hemodynamic instability during TAVR can be triggered by several factors, including anesthesia, RVP, severe bleeding, as well as conduction and rhythm disturbances.

RVP is implemented to ensure transient cardiac standstill for valve positioning and deployment, as well as for pre- and postdilatation to minimize the risk of valve dislodgement. It diminishes cardiac output leading to severe hypotension. Bagur et $\mathrm{al}^{28}$ investigated the influence of the number of procedural RVP runs on the development of AKI following TAVR and found no significant correlation. In turn, Fefer et $\mathrm{al}^{29}$ proved the opposite. They reported that having three or more rapid pacing episodes significantly increases AKI rate to $28 \%$ from $18 \%$ observed in patients undergoing less than three runs of pacing. Interestingly, the latter authors also suggested that AKI can not only result from hemodynamic imbalance on pacing but also from the presence of cardiac injury revealed by a more pronounced elevation in myocardial biomarkers. In the light of the available data on PVR and its possible relation not only with AKI but also with stroke incidence, operators should aim to minimize its use. ${ }^{29}$

Furthermore, the role of intraoperative hypotension is also mirrored by the results of studies demonstrating an even ninefold increase in AKI risk within the post-TAVI use of an intra-aortic balloon pump. ${ }^{30}$

\section{Embolization}

Embolization is more a speculative rather than a scientifically proved cause of AKI. It is an attractive pathophysiologic explanation based on profound research in the field of postTAVR neurologic complication. These studies show that the procedure is accompanied by a great burden of embolic debris, thereby potentially also harming the kidneys. ${ }^{31}$ 


\section{Access route}

Studies and registries have displayed an association between the non-transfemoral approach and the higher rates of AKI compared to the transfemoral route. This was confirmed in an extensive meta-analysis by Thongprayoon et al ${ }^{32}$ with data from over 5,000 patients. Interestingly, when it comes to severe AKI, the authors found no differences between the groups. On the other hand, the UK TAVI registry reported a correlation between the risk of new dialysis requirement and the non-transfemoral route. ${ }^{4}$ There are a few explanations for the phenomenon of higher AKI rates via the nonfemoral access. First of all, this type of access is generally a secondline option dedicated for patients with small, severely calcified and/or tortuous arteries in the setting of severely diffuse atherosclerosis that is already an independent predictor of AKI. Secondly, the apical access still requires wire manipulation in the aorta and thus poses a risk of embolization. Finally, the non-transfemoral route requires general anesthesia, which has been widely replaced with sedation in the case of the femoral access.

\section{Postprocedural}

\section{Paravalvular aortic regurgitation}

Paravalvular aortic regurgitation is a common complication of TAVR and is seen at a much higher rate after TAVR than after conventional surgery. ${ }^{1}$ It can affect up to $70 \%$ of patients and up to $24 \%$ when limited to a moderate or severe degree. ${ }^{33}$ Hopefully, new-generation valves are more flexible to correct if malpositioned and thus associated with a lower burden of paravalvular aortic regurgitation. ${ }^{34}$ Data demonstrate a relation of at least moderate-degree paravalvular regurgitation with increased AKI incidence, dialysis requirement, as well as short- and long-term mortality., ${ }^{43}$ In their Doppler-based renal studies, Sinning et al gave a mechanistic explanation to this association. They demonstrated that the presence of aortic regurgitation is related to significantly increased renal resistance index (RRI) due to the reduced systemic diastolic pressure and increased pulse pressure in kidney arteries. ${ }^{34}$ As a result, the kidneys are underperfused over the diastole and develop the injury.

\section{Outcomes}

\section{Renal replacement therapy}

RRT is a direct consequence of AKI associated with high short- and long-term mortality, a decrease in quality of life, and an increase in the costs of treatment. The prevalence of AKI requiring RRT varies between $0 \%$ and $21 \%$, and the discrepancy is owed to the fact that many studies exclude patients with advanced CKD. ${ }^{4} \mathrm{~A}$ recent meta-analysis dedicated to assessing the impact of AKI after TAVR reported the rate of new RRT of $5.8 \% .{ }^{36}$ Of note, data from the UK TAVI registry show that the proportion of patients requiring RRT is trending down over time from $6.1 \%$ in the years 2007-2008 to 2.3\% in 2013-2014 independent of patient comorbidities. ${ }^{4}$ Among factors contributing to such an improvement, the authors outline experience in the procedure, patient qualification, and peri- and post-procedural care optimization. When compared to SAVR patients, the rates of RRT in TAVR patients are comparable, based on the data from a recent meta-analysis of randomized trials. ${ }^{37}$ However, in studies limited to patients with advanced CKD ( $\geq$ stage 4 ), patients undergoing TAVR seem to require less RRT compared to those undergoing SAVR. ${ }^{20}$

\section{Mortality}

The occurrence of post-TAVR AKI is related to significantly higher short- and long-term mortality. The 30-day mortality rate for patients with AKI following TAVR ranges from $7.8 \%$ to $29 \%$ compared to $2 \%$ to $15 \%$ for those without AKI. ${ }^{38}$ Similarly, after SAVR complicated with AKI, the 30 -day mortality rate is between $5.5 \%$ and $46 \%$, which is up to eightfold higher compared to SAVR free of AKI. ${ }^{37}$ Furthermore, post-TAVR AKI confers up to a fourfold increase in 1-year mortality, and the impact is still observed in a 3-year follow-up. ${ }^{39}$

\section{Cognitive impairment}

Cognitive impairment is prevalent among patients scheduled for TAVR. Its etiology is complex and encompasses, among many other, atherosclerosis, embolism, hypertension, and CKD. The link between CKD and cognitive decline is widely acknowledged; however, little is known about the impact of AKI ${ }^{40}$ Our own data suggest that periprocedural kidney insult can contribute to the deterioration of cognitive functions in TAVR patients with beta- 2 microglobulin playing the key role in this kidney-brain interaction..$^{41}$ For this elderly population, life extension can be at least as essential as maintaining mental independence; thus, the phenomenon is worth attention.

\section{Prevention}

Unfortunately, there is no consensus on AKI prevention in TAVR patients. Currently, a reasonable patient-tailored approach focused on limiting risk factors is advised. ${ }^{13} \mathrm{We}$ have already learnt from cardiac surgery experience that the introduction of "KDIGO bundle" can be beneficial. ${ }^{41}$ 
Moreover, the intervention with the supportive care "bundle" should encompass optimization of 1) volume and hemodynamic parameters, which in the case of TAVR means volume optimization before the procedure, reduction of PVR, bleeding, and vascular complications; 2) restriction of nephrotoxic drugs, mainly contrast agents; and 3) prevention of hypoglycemia. Experience and technologic advancement help to reduce AKI rates, which was proved in the meta-analysis by Ando et al in their outcomes comparison between old-generation (Sapien, Sapien XT, or CorValve) and new-generation valves (ie, Engager, Portico, Sapien 3). ${ }^{42}$ The authors attributed these results mainly to the decrease in bleeding and vascular complications related to the new valve design and the smaller sheath size. Furthermore, they found a significant depletion of paravalvular regurgitation with new-generation valves, which they suggest is due to the fact that replacement of malpositioned device became feasible. ${ }^{34}$

There are also promising tools on the horizon, which can help in either the prevention or early detection of AKI after TAVR. The previously mentioned RenalGuard System is an example of the former. Meanwhile, Doppler-based RRI measurements can be useful in early AKI detection. Sinning et al proved that RRI was able to indicate AKI in the clinically crucial early hours immediately following the TAVR procedure, which was earlier than the rise in either creatinine or cystatin C. ${ }^{35}$ Although encouraging, both devices await their broad acceptance in everyday practice.

Last but not the least, discussing prevention, a phenomenon reverse to AKI, acute kidney recovery (AKR) after TAVR, needs to be mentioned. It appears that an uncomplicated relief of severe AS by an improvement in hemodynamic parameters can lead to an acute recovery of kidney function. It has already been reported in patients who underwent left ventricular assist device implantation. ${ }^{43}$ Currently, there is no clear definition of AKR. The dual definition by Azarbal et al mirrors AKI as based on 1) a $25 \%$ improvement in eGFR over 48 hours after the procedure or 2 ) a decrease of $\geq 0.3 \mathrm{mg} / \mathrm{dL}$ in serum creatinine over 48 hours after TAVR, while Nijenhuis et al refer to the post- to pre-TAVR ratio of serum creatinine of $\leq 0.80$, which significantly affected prevalence amounting to $32 \%, 9 \%$, and $15 \%$, respectively. ${ }^{44,45}$ Interestingly, Nijenhuis et al demonstrated that AKR is associated with shorter hospital stay and, what is of the highest importance, it has a protective effect on 2-year mortality, which was as low as $2.1 \%$ compared to $4.6 \%$ and $44.8 \%$ (both $P<0.01$ ) in a stable creatinine level or AKI group, respectively. ${ }^{45}$ From the pathophysiologic point of view, a successful approach to limit AKI can increase AKR rates; thus, prevention might prove again to be the best treatment.

\section{Conclusion}

In the light of demographic changes in the global population and the advances in interventional cardiology, TAVR era has just started. AKI is a prevalent complication of the procedure affecting mortality and quality of life. The knowledge of risk factors and available preventive measures can help in the appropriate selection of patient, procedure technique, and postprocedural management.

\section{Disclosure}

The authors report no conflicts of interest in this work.

\section{References}

1. Baumgartner H, Falk V, Bax JJ; ESC Scientific Document Group. ESC scientific document group 2017 ESC/EACTS guidelines for the management of valvular heart disease. Eur Heart J. 2017;38(36):2739-2791.

2. Oguri A, Yamamoto M, Mouillet G, et al; FRANCE 2 Registry investigators. Impact of chronic kidney disease on the outcomes of transcatheter aortic valve implantation: results from the France 2 registry. EuroIntervention. 2015;10(9):e1-e9.

3. Najjar M, Salna M, George I. Acute kidney injury after aortic valve replacement: incidence, risk factors and outcomes. Expert Rev Cardiovasc Ther. 2015;13(3):301-316.

4. Ferro CJ, Law JP, Doshi SN, et al; UK TAVI Steering Group and the National Institute for Cardiovascular Outcomes Research. Dialysis following transcatheter aortic valve replacement: risk factors and outcomes. J Am Coll Cardiol Int. 2017;10:240-247.

5. Siddiqui WJ, Alvarez C, Aslam M, et al. Meta-analysis comparing outcomes and need for renal replacement therapy of transcatheter aortic valve implantation versus surgical aortic valve replacement. Am J cardiol. 2018;122(3):468-476.

6. Reinöhl J, Kaier K, Reinecke H, et al. Effect of availability of transcatheter aortic-valve replacement on clinical practice. N Engl J Med. 2015; 373(25):2438-2447.

7. Zhang S, Kolominsky-Rabas PL. How TAVI registries report clinical outcomes-a systematic review of endpoints based on VARC-2 definitions. PLoS One. 2017;12(9): 0180815.

8. Kappetein AP, Head SJ, Généreux P, et al; Valve Academic Research Consortium-2. Updated standardized endpoint definitions for transcatheter aortic valve implantation: the Valve Academic Research Consortium-2 consensus document. J Thorac Cardiovasc Surg. 2013;145(1):6-23.

9. Shacham Y, Rofe M, Leshem-Rubinow E, et al. Usefulness of urine output criteria for early detection of acute kidney injury after transcatheter aortic valve implantation. Cardiorenal Med.2014;4(3-4):155-160.

10. Ostermann M, Joannidis M. Acute kidney injury 2016: diagnosis and diagnostic workup. Crit Care. 2016;20(1):299.

11. Vavilis G, Evans M, Jernberg T, Rück A, Szummer K. Risk factors for worsening renal function and their association with long-term mortality following transcatheter aortic valve implantation: data from the SWEDEHEART registry. Open Heart. 2017;4(2):e000554.

12. Shlipak MG, Mattes MD, Peralta CA. Update on cystatin C: incorporation into clinical practice. Am J Kidney Dis. 2013;62(3):595-603.

13. Zaouter C, Priem F, Leroux L, et al. New markers for early detection of acute kidney injury after transcatheter aortic valve implantation. Anaesth Crit Care Pain Med. 2018;37(4):319-326.

14. Dusse F, Edayadiyil-Dudásova M, Thielmann M, et al. Early prediction of acute kidney injury after transapical and transaortic aortic valve implantation with urinary G1 cell cycle arrest biomarkers. BMC Anesthesiol. 2015;16(1):76.

15. Arsalan M, Ungchusri E, Farkas R, et al. Novel renal biomarker evaluation for early detection of acute kidney injury after transcatheter aortic valve implantation. Proc (Bayl Univ Med Cent). 2018;31(2):171-176. 
16. Załęska-Kocięcka M, Skrobisz A, Wojtkowska I, et al. Serum beta-2 microglobulin levels for predicting acute kidney injury complicating aortic valve replacement. Interact Cardiovasc Thorac Surg. 2017;25(4): 533-540.

17. Otto CM. Valvular aortic stenosis: disease severity and timing of intervention. J Am Coll Cardiol. 2006;47(11):2141-2151.

18. Villablanca PA, Mathew V, Thourani VH, et al. A meta-analysis and metaregression of long-term outcomes of transcatheter versus surgical aortic valve replacement for severe aortic stenosis. Int J Cardiol. 2016;225: 234-243.

19. Kumar N, Khera R, Garg N, et al. Comparison of outcomes of transcatheter versus surgical aortic valve replacement in patients with chronic kidney disease. Am J Cardiol. 2018;121(3):343-348.

20. Doshi R, Shah J, Patel V, Jauhar V, Meraj P. Transcatheter or surgical aortic valve replacement in patients with advanced kidney disease: a propensity score-matched analysis. Clin Cardiol. 2017;40(11):1156-1162.

21. Alqahtani F, Aljohani S, Boobes K, et al. Outcomes of transcatheter and surgical aortic valve replacement in patients on maintenance dialysis. Am J Med. 2017;130(12):1464.e1-1464.e11.

22. Holmes DR Jr, Brennan JM, Rumsfeld JS, et al; STS/ACC TVT Registry. Clinical outcomes at 1 year following transcatheter aortic valve replacement. JAMA. 2015;313(10):1019-1028.

23. Makki N, Lilly SM. Advanced chronic kidney disease: relationship to outcomes post-TAVR, a meta-analysis. Clin Cardiol. 2018;41(8): 1091-1096.

24. Yamamoto M, Hayashida K, Mouillet G, et al. Renal function-based contrast dosing predicts acute kidney injury following transcatheter aortic valve implantation. JACC Cardiovasc Interv. 2013;6(5):479-486.

25. Giannini F, Latib A, Jabbour RJ, et al. The ratio of contrast volume to glomerular filtration rate predicts acute kidney injury and mortality after transcatheter aortic valve implantation. Cardiovasc Revasc Med. 2017;18(5):349-355.

26. Auffret V, Lefevre T, Van Belle E, et al; FRANCE TAVI Investigators. Temporal trends in transcatheter aortic valve replacement in France am coll Cardiol. J Am Coll Cardiol. 2017;70(1):42-55.

27. Barbanti M, Gulino S, Capranzano P, et al. Acute kidney injury with the RenalGuard system in patients undergoing transcatheter aortic valve replacement: the PROTECT-TAVI trial (prophylactic effecT of furosEmide-induCed diuresis with matched isotonic intravenous hydraTion in transcatheter aortic valve implantation). JACC Cardiovasc Interv. 2015;8(12):1595-1604.

28. Bagur R, Webb JG, Nietlispach F, et al. Acute kidney injury following transcatheter aortic valve implantation: predictive factors, prognostic value, and comparison with surgical aortic valve replacement. Eur Heart J. 2010;31(7):865-874.

29. Fefer P, Bogdan A, Grossman Y, et al. Impact of rapid ventricular pacing on outcome after transcatheter aortic valve replacement. J Am Heart Assoc. 2018;7(14):e009038.

30. Thongprayoon C, Cheungpasitporn W, Srivali N, Kittanamongkolchai W, Greason KL, Kashani KB. Incidence and risk factors of acute kidney injury following transcatheter aortic valve replacement. Nephrology. 2016;21(12):1041-1046.
31. Van Mieghem NM, El Faquir N, Rahhab Z, et al. Incidence and predictors of debris embolizing to the brain during transcatheter aortic valve implantation. JACC Cardiovasc Interv. 2015;8(5):718-724.

32. Thongprayoon C, Cheungpasitporn W, Gillaspie EA, Greason KL, Kashani KB. The risk of acute kidney injury following transapical versus transfemoral transcatheter aortic valve replacement: a systematic review and meta-analysis. Clin Kidney J. 2016;9(4):560-566.

33. Généreux P, Head SJ, Hahn R, et al. Paravalvular leak after transcatheter aortic valve replacement: the new Achilles' heel? A comprehensive review of the literature. J Am Coll Cardiol. 2013;61(11):1125-1136.

34. Ando T, Takagi H, Briasoulis A, Grines CL, Afonso L. Comparison of health related quality of life in transcatheter versus surgical aortic valve replacement: a meta-analysis. Heart Lung Circ. 2018;pii: S1443-9506(18): 31841-31849.

35. Sinning JM, Adenauer V, Scheer AC, et al. Doppler-based renal resistance index for the detection of acute kidney injury and the non-invasive evaluation of paravalvular aortic regurgitation after transcatheter aortic valve implantation. EuroIntervention. 2014;9(11):1309-1316.

36. Gargiulo G, Sannino A, Capodanno D, et al. Impact of postoperative acute kidney injury on clinical outcomes after transcatheter aortic valve implantation: a meta-analysis of 5,971 patients. Cathet Cardiovasc Intervent. 2015;86(3):518-527.

37. Siddiqui WJ, Alvarez C, Aslam M, et al. Meta-analysis comparing outcomes and need for renal replacement therapy of transcatheter aortic valve implantation versus surgical aortic valve replacement. Am J Cardiol. 2018;122(3):468-476.

38. Ram P, Mezue K, Pressman G, Rangaswami J. Acute kidney injury post-transcatheter aortic valve replacement. Clin Cardiol. 2017;40(12) 1357-1362.

39. Arsalan M, Squiers JJ, Farkas R, et al. Prognostic usefulness of acute kidney injury after transcatheter aortic valve replacement. Am J Cardiol. 2016;117(8):1327-1331

40. Bugnicourt JM, Godefroy O, Chillon JM, Choukroun G, Massy ZA. Cognitive disorders and dementia in CKD: the neglected kidney-brain axis. J Am Soc Nephrol. 2013;24(3):353-363.

41. Załęska-Kocięcka M, Jezierski P, Grabowski M, et al. Role of $\beta 2$-microglobulin in postoperative cognitive decline. Biomark Med. 2017;11(3):245-253.

42. Meersch M, Schmidt C, Hoffmeier A, et al. Prevention of cardiac surgery-associated AKI by implementing the KDIGO guidelines in high risk patients identified by biomarkers: the PrevAKI randomized controlled trial. Intensive Care Med. 2017;43(11):1551-1561.

43. Butler J, Geisberg C, Howser R, et al. Relationship between renal function and left ventricular assist device use. Ann Thorac Surg. 2006; 81(5):1745-1751.

44. Azarbal A, Leadholm KL, Ashikaga T, Solomon RJ, Dauerman HL. Frequency and prognostic significance of acute kidney recovery in patients who underwent transcatheter aortic valve implantation. Am J Cardiol. 2018;121(5):634-641.

45. Nijenhuis VJ, Peper J, Vorselaars VMM, et al. Prognostic value of improved kidney function after transcatheter aortic valve implantation for aortic stenosis. Am J Cardiol. 2018;121(10):1239-1245.
Clinical Interventions in Aging

\section{Publish your work in this journal}

Clinical Interventions in Aging is an international, peer-reviewed journal focusing on evidence-based reports on the value or lack thereof of treatments intended to prevent or delay the onset of maladaptive correlates of aging in human beings. This journal is indexed on PubMed Central, MedLine,
CAS, Scopus and the Elsevier Bibliographic databases. The manuscript management system is completely online and includes a very quick and fair peer-review system, which is all easy to use. Visit http://www.dovepress. com/testimonials.php to read real quotes from published authors. 